\title{
IDENTIFICACIÓN IN SILICO DEL MIMETISMO MOLECULAR ENTRE EPITOPES T DE Neisseria meningitidis $B$ Y EL PROTEOMA HUMANO
}

\author{
Alexander Batista-Duharte ${ }^{1, a}$, Bruno Téllez, ${ }^{2, b}$, Maybia Tamayo ${ }^{1, c}$, Deivys Portuondo ${ }^{1, d}$, Osmir Cabrera ${ }^{3, e}$, \\ Gustavo Sierra ${ }^{3, f}$, Oliver Pérez ${ }^{3,9}$
}

\section{RESUMEN}

El objetivo del estudio fue determinar los epítopes $T$ de cuatro de las proteínas antigénicas más frecuentes de la membrana externa de Neisseria meningitidis B e identificar los sitios más relevantes donde existe mimetismo molecular para estos epítopes en seres humanos. Para ello se realizó un estudio in silico (estudios que usan herramientas bioinformáticas) usando las bases de datos SWISS-PROT/TrEMBL SYFPEITHI y FASTA, las cuales se emplearon para la determinación de las secuencias proteicas, la predicción de los epítopes T CD4 y CD8, y la determinación del mimetismo molecular en humanos, respectivamente. Se encontró similitud molecular en varias proteínas humanas presentes en diferentes órganos y tejidos, entre ellos: hígado, piel y epitelios, cerebro, sistema linfático y testículos, destacando las encontradas en estos últimos, ya que ellas mostraron la frecuencia más alta de secuencias miméticas. Este hallazgo ayuda a comprender el éxito de $N$. meningitidis $\mathrm{B}$ para colonizar tejidos humanos, el fracaso de ciertas vacunas contra esta bacteria e incluso ayuda a explicar posibles reacciones autoimmunes asociadas a la infección o vacunación.

Palabras clave: Mimetismo molecular; Vacunas; Autoinmunidad; Bioinformática; Neisseria meningitidis sorogrupo B (fuente: DeCS BIREME).

\section{IN SILICO IDENTIFICATION OF MOLECULAR MIMICRY BETWEEN T-CELL EPITOPES OF Neisseria meningitidis B AND THE HUMAN PROTEOME}

\section{ABSTRACT}

\begin{abstract}
The objective of the study was to determine the T-cell epitopes of four of the most frequent antigenic proteins of the outer membrane of Neisseria meningitidis B, and to identify the most relevant sites for molecular mimicry with T-cell epitopes in humans. In order to do so, an in silico study -a type of study that uses bioinformatic tools- was carried out using SWISS-PROT/TrEMBL, SYFPEITHI and FASTA databases, which helped to determine the protein sequences, CD4 and CD8 T-cell epitope prediction, as well as the molecular mimicry with humans, respectively. Molecular similarity was found in several human proteins present in different organs and tissues such as: liver, skin and epithelial tissues, brain, lymphatic system and testicles. Of these, those found in testicles were more similar, showing the highest frequency of mimetic sequences. This finding shed light on the success of $N$. meningitidis $\mathrm{B}$ to colonize human tissues and the failure of certain vaccines against this bacterium, and it even helps to explain possible autoimmune reactions associated with the infection or vaccination.
\end{abstract}

Key words: Molecular mimicry; Vaccines; Autoimmunity; Bioinformatics; Neisseria meningitidis, serogroup B (source: MeSH NLM).

\section{INTRODUCCIÓN}

La Neisseria meningitidis es uno de los principales agentes causales de meningoencefalitis y septicemia en todo el mundo. Para la prevención de esta enfermedad, existen diversas vacunas basadas en conjugados de polisacáridos capsulares contra los serogrupos $A$, C, W-135 e Y. En cambio, la obtención de vacunas polisacarídicas contra el serogrupo $B$ no ha tenido éxito debido a la pobre inmunogenicidad del polisacárido B y la similitud estructural con glicoproteínas humanas, lo cual ha movilizado el interés por la obtención de vacunas a base de antígenos proteicos contenidos en la membrana externa ${ }^{(1)}$. Varios antígenos proteicos mayores, abundantes en la membrana de $N$. meningitidis, cumplen un importante papel en la patogénesis de la enfermedad, así como en la inducción de una respuesta inmune protectora ${ }^{(2)}$, por lo que se tienen en cuenta

\footnotetext{
Centro de Toxicología y Biomedicina, Universidad de Ciencias Médicas de Santiago de Cuba. Santiago de Cuba, Cuba.

Facultad de Ciencias Naturales, Departamento de Biología, Universidad de Oriente. Santiago de Cuba, Cuba.

Instituto Finlay. Ciudad Habana, Cuba.

Médico inmunólogo, doctor en Ciencias Médicas; b licenciado en Biología; ' licenciada en Farmacia, máster en Biotecnología; dicenciado en Biología, máster en Biotecnología; ${ }^{\mathrm{e}}$ doctor en Ciencias de la Salud; ${ }^{\mathrm{f}}$ médico inmunólogo, doctor en Ciencias Médicas; ${ }^{\mathrm{g}}$ médico inmunólogo, doctor en Ciencias Médicas Recibido: 26-12-12 Aprobado: 22-05-13

Citar como: Batista-Duharte A, Téllez B, Tamayo M, Portuondo D, Cabrera O, Sierra G, et al. Identificación in silico del mimetismo molecular entre epitopes T de Neisseria meningitidis B y el proteoma humano. Rev Peru Med Exp Salud Publica. 2013;30(3):441-5.
} 
durante la formulación de vacunas. Algunos de estos antígenos, como el PorB, además de tener propiedades antigénicas ejercen una importante acción como adyuvante a través de la interacción con receptores de la inmunidad innata como los receptores tipo Toll 1 y 2 (TLR-1 y TLR-2), reforzando su interés para la obtención de candidatos vacunales ${ }^{(3)}$.

En Cuba, el empleo del preoteoliposoma de $N$. meningitidis $B$, como base estructural de la formulación VA-MENGOC-BC, ha constituido la primera vacuna exitosa contra este serogrupo ${ }^{(4)}$. Varias proteínas mayoritarias de la vesícula de membrana externa (VME) de $N$. meningitidis, son componentes esenciales de VA-MENGOC-BC y de sus congéneres AFPL1 (Adyuvante Finlay Proteoliposoma 1) y AFCo1 (Adjuvante Finlay Cocleato 1) ${ }^{(5)}$.

Por otro lado, las reacciones autoinmunes constituyen parte de las manifestaciones inmunotóxicas de las vacunas adyuvadas ${ }^{(6)}$. Numerosos reportes de posibles asociaciones entre determinadas vacunas y fenómenos autoinmunes, así como varias evidencias en biomodelos experimentales sustentan esa posibilidad, en la que están involucrados, además de la potente inmunoestimulación, el mimetismo molecular y la susceptibilidad genética ${ }^{(7)}$.

La evaluación del riesgo de desarrollar una respuesta autoinmune en humanos es difícil, debido a la complejidad etiológica de estas enfermedades, y a la carencia de modelos animales apropiados para estudiar el impacto de la vacunación en la inducción o exacerbación de estas enfermedades. Sin embargo, los análisis bioinformáticos (in silico) pueden ofrecer valiosas informaciones acerca de la similitud secuencial o conformacional con estructuras propias que puedan teóricamente inducir el desarrollo de una respuesta autoinmune, por lo que pueden servir de herramientas para la selección de candidatos antigénicos más eficaces y seguros ${ }^{(8,9)}$. El objetivo del presente estudio fue determinar los epítopes $T$ de cuatro de las proteínas antigénicas más frecuentes expresadas en la membrana externa de $N$. meningitidis $B$ e identificar los sitios más relevantes donde existe mimetismo molecular para estos epítopes en seres humanos.

\section{EL ESTUDIO}

Se realizó un estudio in silico en el Centro de Toxicología y Biomedicina de Santiago de Cuba, en el año 2010. El estudio constó de tres etapas: En la primera de ellas se determinó la secuencia aminoacídica de las cuatro proteínas antigénicas más frecuentemente expresadas en la membrana externa de $N$. meningitidis $B$ (PorB, $\mathrm{HmbR}$, FrpB y OpC) para lo cual se empleó el programa
UniProtKB/Swiss-Prot/TrEMBL, en la segunda etapa se determinaron los epítopes T CD4 y CD8 con el programa SYFPEITHI, y en la tercera etapa se determinaron las localizaciones tisulares de proteínas con mimetismo molecular para epítopes T CD8 y proteoma humano para lo cual se empleó el programa FASTA.

\section{DETERMINACIÓN DE SECUENCIAS PROTEICAS:}

Las secuencias de aminoácidos se determinaron a través de la base de datos UniProtKB/Swiss-Prot usando como descriptores el nombre del antígeno proteico mayor de Neisseria meningitidis serogrupo B CU385. Las secuencias seleccionadas fueron: F0AV50_NEIME, con 935597 [NCBI] como identificador taxonómico para PorB; FOARR9_NEIME, 935597 [NCBI] para HmbR; Q9JXL3_NEIMB, 491 [NCBI] para FrpB; y Q7DDI3_ NEIMB 491 [NCBI] para OpC.

\section{LA PREDICCIÓN DE EPÍTOPES T CD4 Y CD8}

Para la predicción de epítopes T CD4 y CD8, se empleó la base de datos SYFPEITHI, que contiene ligandos del complejo mayor de histocompatibilidad $(\mathrm{CMH})$ y motivos peptídicos relacionados $\mathrm{A}$ partir de la secuencia primaria de cada una de las proteínas se obtuvieron péptidos de nueve residuos de extensión (nonámeros) que pueden ser presentados por moléculas del CMH clase I, particularmente la molécula HLA-A2, y ser reconocidos por linfocitos T CD8 humanos.

En la sección "predicción de epitopes" de la base de datos SYFPEITHI, se seleccionó la molécula HLA-A*02:01, se fijó la cantidad de nueve aminoácidos como longitud de los péptidos a generar y se introdujo la secuencia de trabajo. Se tomaron los primeros nonámeros con una puntuación igual o mayor que 20, teniendo en cuenta que el algoritmo de trabajo de SYFPEITHI, asigna como puntuación máxima 36 para un $80 \%$ de confiabilidad. Los nonámeros con puntuación más alta serán los epitopes naturales más probables. Se determinó si algunos de esos péptidos $u$ otros similares han sido reportados previamente y se encuentran en la colección contenida en la base de datos SYFPEITHI. De la misma secuencia primaria se obtuvieron también péptidos de quince residuos de extensión con afinidad a las moléculas del $\mathrm{CMH}$, especificamente HLA-DRB1*01:01, y que puedan ser reconocidos por linfocitos T CD4 humanos.

\section{DETERMINACIÓN DE MIMETISMO MOLECULAR EN HUMANOS}

El algoritmo FASTA se utilizó para detectar los sitios anatómicos que contienen secuencias proteicas miméticas con los nonámeros seleccionados. Para 
Tabla 1. Mimetismo entre proteínas humanas y proteínas de Neisseria meningitidis B

\begin{tabular}{|c|c|c|c|c|}
\hline $\begin{array}{l}\text { Proteína de } N \text {. } \\
\text { meningitidis } B\end{array}$ & Función & Nonámeros & Proteínas humanas con mimetismo & Localización tisular \\
\hline \multirow[t]{2}{*}{ PorB (331 aa) } & \multirow{2}{*}{$\begin{array}{l}\text { Porina. Permite } \\
\text { el paso de iones } \\
\text { a través de la } \\
\text { membrana. Define los } \\
\text { serotipos }\end{array}$} & $\begin{array}{l}\text { DLGNGLKAI } \\
\text { VAAMADVTL } \\
\text { GIVDLGSKI }\end{array}$ & $\begin{array}{l}\text { Proteína integral de membrana CII-3b } \\
\text { 1-Acil-sn-glicerol-3-fosfato aciltransferasa ganma } \\
\text { LFIRE } 1\end{array}$ & \multirow{2}{*}{$\begin{array}{l}\text { Sangre } \\
\text { Piel, testículos, hígado fetal } \\
\text { Hígado fetal } \\
\text { Hígado, testículos }\end{array}$} \\
\hline & & LRVGRLNSV & PRO0907 & \\
\hline $\mathrm{HmBr}(786$ aa) & $\begin{array}{l}\text { Es un sideróforo, } \\
\text { mediante el cual } N \text {. } \\
\text { meningitidis adquiere } \\
\text { el hierro procedente } \\
\text { de la hemoglobina }\end{array}$ & $\begin{array}{l}\text { TLRAGVYNL } \\
\text { QLGGGRHRL } \\
\text { LLDDRQFGV } \\
\text { SLGYAKSKL }\end{array}$ & $\begin{array}{l}\text { Peroxisomal trans-2-enoil CoA reductasa } \\
\text { FBXL18 } \\
\text { Glicoproteina de membrana neuronal M6-a } \\
\text { Proteina pseudo asparaginasa }\end{array}$ & $\begin{array}{l}\text { Hígado, hígado fetal, } \\
\text { placenta } \\
\text { Pulmones, testículos, } \\
\text { cerebro, medula espinal } \\
\text { Corteza cerebral } \\
\text { Testículos }\end{array}$ \\
\hline FrpB (714 aa) & $\begin{array}{l}\text { También conocida } \\
\text { como FetA. Permite } \\
\text { que } N \text {. meningitidis } \\
\text { adquiera el hierro } \\
\text { procedente de la } \\
\text { hemoglobina }\end{array}$ & $\begin{array}{l}\text { KIRTNIVTL } \\
\text { SLLSLTLAA } \\
\text { HAAENNAKV } \\
\text { ILYHQGRFI } \\
\text { AIITKTVDA }\end{array}$ & $\begin{array}{l}\text { Proteina Ras (Rab-25) } \\
\text { Proteína Treacle } \\
\text { Proteína transmenbrana } 179 \\
\text { Proteína C2orf31 } \\
\text { Familia NUAK, SNF1 quinasa } 2 \\
\text { Dominio Coiled-coil conteniendo proteina } 79\end{array}$ & $\begin{array}{l}\text { Intestinos, ovarios, páncreas, } \\
\text { piel, testículos. } \\
\text { Ojos, piel, epitelios. } \\
\text { Cerebelo } \\
\text { Testículos } \\
\text { Testículos, sistema linfático. } \\
\text { Testículos }\end{array}$ \\
\hline OpC (272 aa) & $\begin{array}{l}\text { Adhesina involucrada } \\
\text { en la adherencia de } \\
N \text {. meningitidis a la } \\
\text { superficie epitelial }\end{array}$ & SIREYGLRV & Proteina IMAA & Sistema linfático \\
\hline
\end{tabular}

aa: amino ácidos

ello se seleccionó la base de datos de proteoma de Homo sapiens. Se clasificaron y cuantificaron las moléculas obtenidas como proteínas de expresión en los diferentes tejidos, desechándose los datos de proteínas hipotéticas (que son aquellas que genera la base de datos por traducción automática de probables secuencias nucleotídicas codificadoras), los fragmentos y los precursores. Luego se procedió a localizar anatómicamente cada una de las proteínas siguiendo el hipervínculo que brinda FASTA en la tabla de resultados donde se muestra la descripción de cada proteína.

\section{HALLAZGOS}

El estudio in silico incluyó cuatro proteínas antigénicas de la membrana de $N$. meningitidis $B$ (PorB, HmbR, FrpB y $\mathrm{OpC}$ ). La función de cada una ellas así como los nonámeros obtenidos a partir de ellas y las proteínas humanas con las que guardan mimetismo, y su localización tisular se muestran en la Tabla 1. Se encontró que la PorB se encontraba en cuatro secuencias con mimetismo localizados en sangre, piel, testículos hígado fetal y adulto; en el caso de $\mathrm{HmbR}$ se encontraron cuatro secuencias presentes en hígado fetal, placenta, pulmones, médula espinal, cerebro y testículos. La proteína FrpB tuvo la mayor cantidad de secuencias miméticas, localizadas en intestinos, ovarios, páncreas, piel, testículos, ojos, piel, epitelios, cerebelo y sistema linfático. De todos estos órganos, el testículo estuvo presente en tres de las cuatro secuencias miméticas. Para el caso de la proteína OpC solo presentó mimetismo en una proteína del sistema linfático. La Figura 1 ilustra el número de proteínas, distribuidas por órganos, con mimetismo molecular entre las cuatro proteínas mayoritarias de $N$. meningitidis $B$ evaluadas y el proteoma humano, observándose que el testículo fue el órgano que presentó mayor número de proteínas miméticas en comparación con el resto de los órganos.

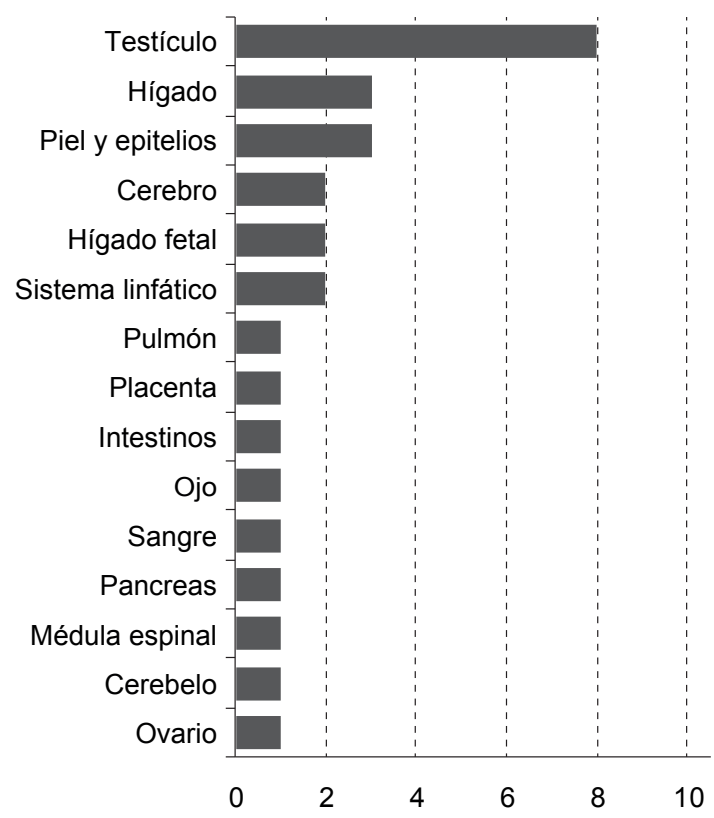

Figura 1. Número de proteínas con mimetismo molecular entre epítopes T de Neisseria meningitidis B y el proteoma humano 


\section{DISCUSIÓN}

La determinación in silico del mimetismo molecular se propone como parte de los ensayos preclínicos toxicológicos de vacunas ${ }^{(9)}$, debido a su papel como uno de los componentes del mosaico de autoinmunidad (10). Fujinami y Oldstone inmunizaron conejos con péptidos derivados de la polimerasa del virus de la hepatitis $\mathrm{B}$, que contiene una secuencia mimética con proteína básica de mielina, y demostraron que es posible la ocurrencia de daños autoinmunes posvacunación (11). A pesar de este y otros reportes experimentales de reacciones autoinmunes inducidas por la inoculación de péptidos miméticos, todavía no se conoce con exactitud su verdadero papel en la inducción de autoinmunidad posvacunal.

En este estudio se evaluó el mimetismo molecular (epitopes T) entre cuatro proteínas mayores de $N$. meningitidis $\mathrm{B}$ presentes en diversas vacunas contra esta bacteria y el proteoma humano. Debido a la gran diversidad en los HLA humanos, se realizó una selección de ellos, basados en la alta frecuencia en la población cubana y en otros países de los haplotipos HLA-A* 0201 y HLA-DRB $1^{*} 01^{(12,13)}$, en tanto existen reportes de que el HLA-DRB1*01 está asociado con fenómenos autoinmunes por adyuvantes ${ }^{(14)}$.

La cantidad de nonámeros generados estuvo en dependencia de la cantidad de aminoácidos en cada una de las secuencias de trabajo. Por no contar con la información suficiente relacionada con la capacidad de inducción de fenómenos autoinmunes por parte de estas proteínas y para aumentar el valor predictivo, se decidió llevar hasta diez los nonámeros correspondientes a las proteínas $\mathrm{OpC}$ y Por $\mathrm{B}$, mientras que las proteínas HmbR y FrpB, por ser de mayor tamaño, se llevaron hasta veinte nonámeros.

En nuestro estudio se detectaron similitudes secuenciales entre las proteínas estudiadas y otras presentes en diversos tejidos humanos, sin incluir las proteínas hipotéticas generadas automáticamente, sino aquellas que han sido estudiadas en el proteoma humano, para lograr un mayor acercamiento a la realidad. De este resultado lo más significativo fue la alta frecuencia de mimetismo en proteínas testiculares superando en tres veces la frecuencia de los otros sitios. Las células germinativas masculinas a pesar de estar protegidas por la barrera hematotesticular ${ }^{(15)}$, con frecuencia son afectadas por una variedad de agentes ambientales y fármacos que interfieren en el equilibrio existente entre supervivencia y apoptosis durante la espermatogénesis. En el caso de las vacunas, se han reportado sospechas de asociación entre vacunación antiparotiditis y orquitis, con un posible mecanismo autoinmune como base ${ }^{(16)}$.
Además, existen reportes de que bajo condiciones de potente inmunoestimulación puede haber una elevación de citocinas proinflamatorias y de una polarización hacia Th1 en testículos, ocasionando una respuesta inflamatoria local que puede conllevar a destrucción de los espermatozoides o, más raramente, a una orquitis autoinmune ${ }^{(17,18)}$. También se puede inducir orquitis autoinmune experimental inmunizando ratas o ratones con homogenatos de testículos en adyuvantes, o por transferencia de linfocitos T CD4+ de animales sensibilizados a animales no inmunizados, incluso se ha detectado experimentalmente trastornos de la fertilidad por inmunización con pequeños péptidos ${ }^{(17,18)}$. Estos reportes pudieran orientar que existe un peligro potencial de afectación testicular posvacunación. Los otros órganos que tuvieron mimetismo molecular con antígenos de $N$. meningitidis $\mathrm{B}$, la frecuencia de mimetismo fue mucho menor, e incluso en la sangre, los tejidos linfáticos y tejidos fetales es poco probable la existencia de efectos autoinmunes ya que en estas regiones los mecanismos de tolerancia son particularmente efectivos ${ }^{(10)}$.

A pesar de estos hallazgos, hasta el momento no existen reportes de eventos autoinmunes asociados con vacunas antimengocócicas. Por otro lado, el mimetismo molecular constituye un mecanismo que emplea $N$. meningitidis para escapar de la respuesta inmune ${ }^{(19)}$. Por ejemplo, el mimetismo entre homopolímeros de ácido siálico capsulares del serogrupo B y un componente de NCAM (del inglés: neural cell-adhesion molecule), presente en el sistema nervioso, propicia la pobre respuesta inmune generada contra la cápsula de este serogrupo, lo que ha dificultado la obtención de vacunas polisacarídicas contra este serogrupo. Así que, el mimetismo molecular en las secuencias de aminoácidos también podría contribuir al éxito en la colonización e invasividad de $N$. meningitidis $B^{(2,19)}$. Sin embargo, la vacuna antimengocócica VA-MENGOC-BC induce una elevada respuesta inmune protectora, lo cual se debe a la variada composición antigénica junto a adyuvantes naturales contenidos en el propio proteoliposoma bacteriano, formulados con el hidróxido de aluminio ${ }^{(4)}$. Por otro lado, esta vacuna se ha utilizado en millones de personas en varios países, incluyendo varones en los que no se ha detectado trastornos de la fertilidad ni otros eventos autoinmunes, en los estudios de farmacovigilancia realizados ${ }^{(20)}$, No obstante, no existen reportes de estudios de anticuerpos contra espermatozoides y de fertilidad en personas posvacunadas contra $N$. meningitidis $B$, por lo que esto podría ser un tema de investigación futura.

Finalmente, hay que señalar que debido a que las reacciones autoinmunes son multifactoriales en su 
causalidad, estos resultados in silico, por sí solos, no pueden ser considerados un biomarcador general de predicción, por lo que deben ser siempre analizados caso a caso conjuntamente con los factores genéticos, ambientales, condiciones subyacentes, entre otros que conforman la medicina personalizada.

En conclusión, se ha demostrado la existencia de mimetismo molecular entre antígenos de $N$. meningitidis $B$ y estructuras humanas, particularmente en testículos Aún se requieren nuevas investigaciones que profundicen en el papel de estas similitudes moleculares y la inmunopatogenia de este microorganismo, especialmente vinculadas a la colonización de tejidos, el fracaso de ciertas vacunas antimengocócicas de subunidades e incluso su papel en posibles reacciones autoinmunes raras que pudieran estar asociadas a la infección o vacunación.
Agradecimientos: al Dr. Orlando Serrano Barrera del Centro de Genética Médica. Las Tunas, Cuba, por su contribución en el uso de las bases de datos y análisis de los resultados.

Contribuciones de autoría: ABD participó en la concepción y diseño del artículo, el análisis e interpretación de datos y en la redacción del artículo. BTM, DPF y OC participaron de la recolección y obtención de resultados. OC participó con el aporte de material de estudio (secuencias proteicas de vacunas cubanas para comparación). GSG participó en el análisis e interpretación de datos. OP participó en el aporte de material de estudio (Electroforesis en gel de poliacrilamida de Neisseria meningitidis B) y el Análisis e interpretación de datos. Todos los autores aprobaron la versión final de artículo.

Fuente de financiamiento: este estudio fue financiado por el Centro de Toxicología y Biomedicina - TOXIMED (Santiago de Cuba, Cuba).

Conflictos de interés: los autores declaran no tener conflictos de interés

\section{REFERENCIAS BIBLIOGRÁFICAS}

1. Feavers IM, Pizza M. Meningococcal protein antigens and vaccines. Vaccine. 2009;27 Suppl 2:B42-50. doi: 10.1016/j.vaccine.2009.05.001.

2. Hill DJ, Griffiths NJ, Borodina E, Virji M. Cellular and molecular biology of Neisseria meningitidis colonization and invasive disease. Clin Sci (Lond). 2010;118(9):547-64. doi: 10.1042/ CS20090513.

3. Wetzler LM. Innate immune function of the neisserial porins and the relationship to vaccine adjuvant activity. Future Microbiol. 2010;5(5):749-58. doi: $10.2217 / \mathrm{fmb} \cdot 10.41$.

4. Sierra GV, Campa HC, Varcacel NM, Garcia IL, Izquierdo PL, Sotolongo PF, et al. Vaccine against group B Neisseria meningitidis: protection trial and mass vaccination results in Cuba. NIPH Ann. 1991;14(2):195-207

5. Pérez O, Batista-Duharte A, González E, Zayas C, Balboa J, Cuello M, et al. Human prophylactic vaccine adjuvants and their determinant role in new vaccine formulations. Braz J Med Biol Res. 2012 Aug;45(8):681-92.

6. Batista-Duharte A, Lindblad EB, Oviedo-Orta E. Progress in understanding adjuvant immunotoxicity mechanisms. Toxicol Lett. 2011;203(2):97-105. doi: 10.1016/j.toxlet.2011.03.001.

7. Batista-Duharte A. Vacunas y autoinmunidad. Una rara asociación bajo debate. Rev Peru Med Exp Salud Publica. 2012;29(2):265-71.

8. Chandra Sh, Singh D, Singh T. Prediction and characterization of T-cell epitopes for epitope vaccine design from outer membrane protein of Neisseria meningitidis serogroup B. Bioinformation. 2009;5(4):155-61.

9. Garçon N, Segal L, Tavares F, Van Mechelen M. The safety evaluation of adjuvants during vaccine development. The AS04 experience. Vaccine. 2011;29(27):4453-9. doi: 10.1016/j. vaccine.2011.04.046.

10. Ryan KR, Patel SD, Stephens LA, Anderton SM. Death, adaptation and regulation: The three pillars of immune tolerance restrict the risk of autoimmune disease caused by molecular mimicry. J Autoimmun. 2007;29(4):262-71

11. Fujinami RS, Oldstone MB, Amino acid homology between the encephalitogenic site of myelin basic protein and virus: mechanism for autoimmunity. Science. 1985;230(4729):1043-5.

12. Paradoa ML, Middleton D, Acosta A, Sarmiento ME, Leyva J. Genes HLA en una muiestra de población cubana. Vaccimonitor. 2000;9(3):1-5

13. Rodríguez LM, Giraldo MC, García N, Velásquez L, París SC, Álvarez CM, et al. Frecuencias alélicas, genotípicas y haplotípicas HLA-A, HLA-B, HLA-DRB1 en donantes fallecidos, Medellín, Colombia. Biomédica. 2007;27(4):537-47.

14. Shoenfeld Y, Agmon-Levin N. 'ASIA' autoimmune/inflammatory syndrome induced by adjuvants. J Autoimmun. $2011 ; 36(1): 4-8$. doi: $10.1016 / j$. jaut.2010.07.003.

15. Fijak M, Bhushan S, Meinhardt A. The Immune Privilege of the Testis. In:
Krause WKH, Naz RK (eds.). Immune Infertility. Berlin: Springer Verlag; 2009. p. 69-77.

16. Clifford V, Wadsley J, Jenner B, Buttery JP. Mumps vaccine associated orchitis. Evidence supporting a potential immune-mediated mechanism. Vaccine. 2010;28(14):2671-3. doi: 10.1016/j.vaccine.2010.01.007.

17. Jacobo P, Guazzone VA, Theas MS, Lustig L, Testicular autoimmunity. Autoimmun Rev. 2011;10(4):201-4. doi: 10.1016/j.autrev.2010.09.026.

18. Xu W, Zhang $\mathrm{X}$, Chen W, Fok KL, Rowlands DK, Chui YL, et al. Immunization with Bin $1 \mathrm{~b}$ decreases sperm motility with compromised fertility in rats. Fertil Steril. 2010;93(3):952-958.e1. doi: 10.1016/j.fertnstert.2008.10.066.

19. LoH,TangCM,Exley RM.Mechanisms of avoidance of host immunity by Neisseria meningitidis and its effect on vaccine development. Lancet Infect Dis. 2009;9(7):418-27. doi: 10.1016/ S1473-3099(09)70132-X.

20. Ochoa Azze CR, Menéndez Hernández J. Prevención de la enfermedad meningocócica. 1ra ed. La Habana: Finlay Ediciones; 2010.

Correspondencia: Alexander Batista Duharte. Dirección: Centro de Toxicología y Biomedicina (TOXIMED), Autopista Nacional Km 1/2 Código Postal 90400, PO Box 4033, Santiago de Cuba. AP 4033.

Teléfono: +5322643926

Correo electrónico: alexanderbatista@medired. scu.sld.cu 